\title{
A reconstruction theorem for locally moving groups acting on completely metrizable spaces
}

\author{
by
}

\author{
Edmund Ben-Ami (Beer Sheva)
}

\begin{abstract}
Let $G$ be a group which acts by homeomorphisms on a metric space $X$. We say the action of $G$ is locally moving on $X$ if for every open $U \subseteq X$ there is a $g \in G$ such that $g\lceil X \neq \mathrm{Id}$ while $g \uparrow(X \backslash U)=\mathrm{Id}$. We prove the following theorem:

Theorem A. Let $X, Y$ be completely metrizable spaces and let $G$ be a group which acts on $X$ and $Y$ with locally moving actions. If the orbits of the action of $G$ on $X$ are of the second category in $X$ and the orbits of the action of $G$ on $Y$ are of the second category in $Y$, then $X$ and $Y$ are homeomorphic.

A particular case of Theorem A gives a positive answer to a question of M. Rubin and J. van Mill who asked whether $X$ and $Y$ are homeomorphic whenever $G$ is strongly locally homogeneous on $X$ and $Y$.
\end{abstract}

1. Introduction. For a topological space $X$ denote by $\mathcal{H}(X)$ the group of all auto-homeomorphisms of $X$. If $X$ is a regular space and $G$ is a subgroup of $\mathcal{H}(X)$ we call $\langle X, G\rangle$ a space-group pair.

Let $\langle X, G\rangle$ be a space-group pair. If for every nonempty open set $U \subseteq X$ there is some $g \in G \backslash\{\mathrm{Id}\}$ such that $g \uparrow(X \backslash U)=\mathrm{Id}$, then we say that $G$ is locally moving on $X$, or that $\langle X, G\rangle$ is a local movement system. Notice that if $\langle X, G\rangle$ is a local movement system, then $X$ has no isolated points.

THeOREM 1.1. Let $X$ and $Y$ be completely metrizable spaces and $\langle X, G\rangle,\langle Y, H\rangle$ be local movement systems with $\varphi: G \cong H$ ( $\varphi$ is an isomorphism of abstract groups, without the action). Then there are $\widehat{X} \subseteq X, \widehat{Y} \subseteq Y$ and $\tau: \widehat{X} \cong \widehat{Y}$ such that

2010 Mathematics Subject Classification: Primary 54E50; Secondary 22 F05.

Key words and phrases: homeomorphism group, locally moving group, reconstruction, rigidity, rich group action, complete metric space.

This paper is a part of the author's Ph.D thesis which is being prepared under the supervision of Prof. Matatyahu Rubin. 
(1) $\widehat{X}$ is co-meager in $X$ and $\widehat{Y}$ is co-meager in $Y$.

(2) $\widehat{X}$ is invariant under $G$ and $\widehat{Y}$ is invariant under $H$.

(3) $\tau$ induces $\varphi$ in the following sense: $\varphi(g) \mid \widehat{Y}=\tau \circ(g \uparrow \widehat{X}) \circ \tau^{-1}$ for every $g \in G$.

Let $\langle X, G\rangle$ and $\langle Y, H\rangle$ be space-group pairs, and $\varphi: G \cong H, \tau: X \cong Y$ be such that $\varphi(g)=\tau \circ g \circ \tau^{-1}$ for every $g \in G$. Then we say that $\tau$ induces $\varphi$.

The conditions in Theorem 1.1 do not ensure that $X$ and $Y$ are homeomorphic. The following example shows this. Let $\mathbb{P}$ denote the irrational numbers, and let $G$ be the group of all auto-homeomorphisms of $\mathbb{R}$ which take $\mathbb{P}$ onto itself. Clearly $G$ is locally moving on both $\mathbb{R}$ and $\mathbb{P}$, and both $\mathbb{R}$ and $\mathbb{P}$ are completely metrizable.

Let $\langle X, G\rangle$ be a space-group pair. Then for every $x \in X$ the set $\{g(x) \mid g \in G\}$ is called the orbit of $x$ under $G$. We denote it by $G(x)$. The following theorem (Theorem A in the abstract) is a trivial consequence of Theorem 1.1.

TheOREM 1.2. Let $X$ and $Y$ be completely metrizable spaces and $\langle X, G\rangle$, $\langle Y, H\rangle$ be local movement systems with $\varphi: G \cong H$. If for every $x \in X, G(x)$ is of the second category in $X$, and for every $y \in Y, H(y)$ is of the second category in $Y$, then there is a $\tau: X \cong Y$ which induces $\varphi$.

The next corollary is just a special case of Theorem 1.2. It gives a positive answer to a question of M. Rubin and J. van Mill [vM]. Let $\langle X, G\rangle$ be a space-group pair. Then we say that $G$ is strongly locally homogeneous or $S L H$ on $X$ if there is an open base $\mathcal{U}$ for the topology of $X$ such that for every $U \in \mathcal{U}$ and every $x, y \in U$ there is $g \in G$ such that $g \uparrow(X \backslash U)=\mathrm{Id}$ and $g(x)=y$. Whenever $\mathcal{H}(X)$ is SLH on $X$, we say that $X$ is SLH.

Corollary 1.3. Let $X$ and $Y$ be completely metrizable spaces and $\langle X, G\rangle,\langle Y, H\rangle$ be space-group pairs such that $G$ is $S L H$ on $X$ and $H$ is SLH on $Y$. If $\varphi: G \cong H$ then there is $\tau: X \cong Y$ such that $\tau$ induces $\varphi$.

In [vM] J. van Mill constructs two SLH metric spaces $X, Y \subseteq \mathbb{S}^{2}$ which are not homeomorphic, while $\mathcal{H}(X)$ and $\mathcal{H}(Y)$ are isomorphic (in fact $X$ and $Y$ are complementary in $\mathbb{S}^{2}$ and every homeomorphism of one of them is extendable to the entire $\mathbb{S}^{2}$ ). In van Mill's example, neither $X$ nor $Y$ are completely metrizable. Thus the completeness condition imposed on all the spaces we deal with in this paper cannot be dropped even if the groups in question are SLH on the respective spaces. However, in van Mill's example, both the actions of $\mathcal{H}(X)$ on $X$ and of $\mathcal{H}(Y)$ on $Y$ can be extended to actions on the whole $\mathbb{S}^{2}$ (which is the metric completion of both $X$ and $Y$ ). This raises the following question, in which $\bar{X}$ denotes the completion of the metric space $X$. 
Question. Let $X, Y$ be metric spaces and $\langle X, G\rangle,\langle Y, H\rangle$ be spacegroup pairs such that $G$ is $\mathrm{SLH}$ on $X, H$ is $\mathrm{SLH}$ on $Y$, and $\varphi: G \cong H$. Assume further that there is no $X \subsetneq X_{1} \subseteq \bar{X}$ such that every member of $G$ extends to a homeomorphism of $X_{1}$, and the same holds for $Y$ and $H$. Is there a homeomorphism $\tau: X \cong Y$ such that $\tau$ induces $\varphi$ ?

Throughout this paper the notation $\tau: X \cong Y$ indicates that $\tau$ is a homeomorphism between $X$ and $Y$. Similarly, if $G$ and $H$ are groups, then $\varphi: G \cong H$ means that $\varphi$ is an isomorphism between $G$ and $H$. Note that we do not equip $G$ and $H$ with any topology, so $\varphi$ is an isomorphism between abstract groups.

2. Local movement systems. We begin this section by stating M. Rubin's reconstruction theorem. We first need some definitions. Let $\operatorname{Ro}(X)$ denote the set of regular open subsets of the space $X$, that is, the open subsets of $X$ for which $U=\operatorname{int}(\operatorname{cl}(U))$. The Boolean algebra of regular open subsets of $X$ is $\operatorname{Ro}(X)=(\operatorname{Ro}(X) ;+, \cdot,-, 0,1)$, where $U+V:=\operatorname{int}(\operatorname{cl}(U \cup V))$, $U \cdot V:=U \cap V,-U:=\operatorname{int}(X \backslash U), 0:=\emptyset$ and $1:=X$. By writing $\psi: \operatorname{Ro}(X) \cong \operatorname{Ro}(Y)$ we mean that $\psi$ is an isomorphism of Boolean algebras.

Let $g \in \mathcal{H}(X)$. Clearly, $g(V) \in \operatorname{Ro}(X)$ for every $V \in \operatorname{Ro}(X)$. Moreover, the mapping $V \mapsto g(V)$ is an automorphism of $\operatorname{Ro}(X)$, denoted by $g^{\text {Ro }}$. Clearly, the mapping $g \mapsto g^{\mathrm{Ro}}$ is a group homomorphism of $\mathcal{H}(X)$ into Aut $(\operatorname{Ro}(X))$. Notice that for Hausdorff spaces, if $g \neq h \in \mathcal{H}(X)$ then $g^{\mathrm{Ro}} \neq h^{\mathrm{Ro}}$ and thus the mapping $g \mapsto g^{\mathrm{Ro}}$ is a group embedding if the space $X$ is Hausdorff, which is always the case for the spaces we deal with. Hence, by abuse of notation, we do not distinguish between $g^{\text {Ro }}$ and $g$.

Now we are ready to state M. Rubin's reconstruction theorem. Recall that $\langle X, G\rangle$ is a local movement system if $X$ is a regular space and $G$ is locally moving on $X$.

Theorem 2.1 ([u2, Corollary 1.4]). Let $\langle X, G\rangle$ and $\langle Y, H\rangle$ be local movement systems with $\varphi: G \cong H$ ( $\varphi$ is an isomorphism of abstract groups, without the action). Then there is a unique $\psi: \operatorname{Ro}(X) \cong \operatorname{Ro}(Y)$ such that $\varphi(g)=\psi \circ g \circ \psi^{-1}$ for every $g \in G$.

We say that $\psi$ induces $\varphi$ whenever $\varphi(g)=\psi \circ g \circ \psi^{-1}$.

Notice that if we wished to be precise we actually should have written $\varphi(g)^{\mathrm{Ro}}=\psi \circ g^{\mathrm{Ro}} \circ \psi^{-1}$. From now on we will use $g$ instead of $g^{\mathrm{Ro}}$ freely.

Remember that we are interested in finding a homeomorphism between the spaces $X$ and $Y$, thus, a mapping between points in $X$ and points in $Y$. Theorem 2.1 already does most of the work by giving us $\psi$, which is a mapping between regular open sets in $X$ and regular open sets in $Y$. Now, it remains to use $\psi$ and some additional conditions on the actions and the 
spaces to derive the homeomorphism we are interested in. Theorem 2.1 is often applied in this way to show spaces are homeomorphic, in [Ru1] for instance.

Notice that it is not true in general that spaces with isomorphic algebras of regular open sets are homeomorphic. For instance, a space and its StoneCech compactification have the same algebras of regular open sets.

The rest of this section is devoted to presenting some consequences of Theorem 2.1 which will come in handy in Section 3.

For any $g \in \mathcal{H}(X)$ the support of $g$ is $\operatorname{supp}(g)=\{x \in X \mid g(x) \neq x\}$.

Proposition 2.2. Let $\langle X, G\rangle$ and $\langle Y, H\rangle$ be space-group pairs such that $\varphi: G \cong H, \psi: \operatorname{Ro}(X) \cong \operatorname{Ro}(Y)$, and $\psi$ induces $\varphi$ (so for every $g \in G$, $\left.\varphi(g)=\psi \circ g \circ \psi^{-1}\right)$. Then for every $g \in G$ and $U \in \operatorname{Ro}(X), \operatorname{supp}(g) \subseteq U$ iff $\operatorname{supp}(\varphi(g)) \subseteq \psi(U)$.

Proof. We show that if $\operatorname{supp}(g) \subseteq U$ then $\operatorname{supp}(\varphi(g)) \subseteq \psi(U)$. The proof of the other direction is similar. Let $U \in \operatorname{Ro}(X)$ and $\operatorname{suppose} \operatorname{supp}(g) \subseteq U$. First note that $\operatorname{supp}(g)=\bigcup\{V \in \operatorname{Ro}(X) \mid V \cap g(V)=\emptyset\}$ since $X$ is Hausdorff. So, for every $V \in \operatorname{Ro}(X), V \cap g(V)=\emptyset$ implies that $V \subseteq U$. We need to show that for every $V \in \operatorname{Ro}(X), \psi(V) \cap \varphi(g)(\psi(V))=\emptyset$ implies that $\psi(V) \subseteq \psi(U)$.

Since $\psi$ induces $\varphi, \psi(g(V))=\varphi(g)(\psi(V))$. Hence $\psi(V) \cap \varphi(g)(\psi(V))=\emptyset$ implies that $\psi(V) \cap \psi(g(V))=\emptyset$, and since $\psi$ is an isomorphism, $V \cap g(V)=\emptyset$. Hence $V \subseteq U$, and so $\psi(V) \subseteq \psi(U)$.

Let $\langle X, G\rangle$ be a space-group pair and $U \subseteq X$. Then $G|U|=\{g \in G \mid$ $\operatorname{supp}(g) \subseteq U\}$ is the subgroup of all members of $G$ whose support is contained in $U$. For every ultrafilter $\mathcal{U}$ in $\operatorname{Ro}(X)$, let $G_{\mathcal{U}}$ be the subgroup of $G$ generated by $\bigcup_{U \in \mathcal{U}} G|X \backslash U|$. Notice that if $x \in X$ and $x \in \bigcap_{U \in \mathcal{U}} \operatorname{cl}(U)$, then $G_{\mathcal{U}} \subseteq G_{x}$ for $G_{x}=\{g \in G \mid g(x)=x\}$. For any two ultrafilters $\mathcal{U}$ and $\mathcal{V}$ in $\operatorname{Ro}(X)$, let $G_{\mathcal{U}, \mathcal{V}}$ be the subgroup of $G$ generated by $G_{\mathcal{U}} \cup G_{\mathcal{V}}$. The following corollary is a consequence of Proposition 2.2 .

Corollary 2.3. Under the assumptions of Proposition 2.2, for any two ultrafilters $\mathcal{U}$ and $\mathcal{V}$ in $\operatorname{Ro}(X), \varphi\left(G_{\mathcal{U}}\right)=H_{\psi(\mathcal{U})}$ and $\varphi\left(G_{\mathcal{U}, \mathcal{V}}\right)=H_{\psi(\mathcal{U}), \psi(\mathcal{V})}$.

The following corollary of Theorem 2.1 will be very useful later on, but is interesting in its own right, since it states that being a locally moving subgroup is a property which is preserved under group isomorphisms:

Corollary 2.4. Let $\langle X, G\rangle$ and $\langle Y, H\rangle$ be local movement systems with $\varphi: G \cong H$. If $\widehat{G}$ is a subgroup of $G$ which is locally moving on $X$, then $\varphi(\widehat{G})$ is a subgroup of $H$ which is locally moving on $Y$.

Proof. It follows from Theorem 2.1 that there is a $\psi: \operatorname{Ro}(X) \cong \operatorname{Ro}(Y)$ which induces $\varphi$, and thus $\varphi$ and $\psi$ are as in Proposition 2.2. Let $V \in \operatorname{Ro}(Y)$. 
Since $\widehat{G}$ is locally moving on $X$, there is some $g \in \widehat{G}$ such that $g \neq \mathrm{Id}$ and $\operatorname{supp}(g) \subseteq \psi^{-1}(V)$. Hence, $\operatorname{supp}(\varphi(g)) \subseteq \psi\left(\psi^{-1}(V)\right)=V$. Since $g \neq \mathrm{Id}$, also $\varphi(g) \neq \mathrm{Id}$. So, since $\operatorname{Ro}(Y)$ is a base for the topology of $Y$ (since $Y$ is metric and thus regular), $\varphi(\widehat{G})$ is locally moving on $Y$.

Denote the fixed points of $G$ by $\operatorname{Fix}(G)=\{x \in X \mid(\forall g \in G)(g(x)=x)\}$. Recall that a subset $A$ of $X$ is said to be nowhere dense in $X$ if $\operatorname{cl}(A)$ has an empty interior.

Proposition 2.5. For every local movement system $\langle X, G\rangle, \operatorname{Fix}(G)$ is nowhere dense in $X$.

Proof. Let $U$ be a nonempty open subset of $X$. To see that $\operatorname{Fix}(G)$ is not dense in $U$, choose $g \in G \mid U \backslash \backslash\{\operatorname{Id}\}$. Since $X$ is Hausdorff, there is some $V \subseteq U$ such that $g(V) \cap V=\emptyset$, and hence $V \cap \operatorname{Fix}(G)=\emptyset$.

3. A theorem for complete metric spaces. This section is devoted to proving Theorem 1.1 and some of its corollaries. Recall that our starting point is the conclusion of Theorem 2.1. So, given an isomorphism $\psi: \operatorname{Ro}(X) \cong \operatorname{Ro}(Y)$, we wish to find out when there exists a homeomorphism $\tau$ such that $\psi(U)=\{\tau(x) \mid x \in U\}$ for every $U \in \operatorname{Ro}(X)$. Whenever such a $\tau$ exists, we wish to derive it from $\psi$ and $\varphi$. We do so by looking at ultrafilters. For every ultrafilter $\mathcal{U}$ in $\operatorname{Ro}(X)$, we look at $\bigcap_{U \in \mathcal{U}} \operatorname{cl}(U)$, which consists of at most one point since $X$ is Hausdorff. Whenever it equals $\{x\}$ we say that $\mathcal{U}$ defines $x$. Since $\psi$ is an isomorphism of Boolean algebras, it induces a bijection between the ultrafilters in $\operatorname{Ro}(X)$ and those in $\operatorname{Ro}(Y)$. Were it a bijection between the ultrafilters which define some point in $X$ and those which define some point in $Y$, we would have a natural candidate for $\tau$. The proof of Theorem 1.1 is just a refinement of this preliminary suggestion.

Since we are interested in ultrafilters which define a point, the following observation will come in handy. We use $B_{\epsilon}(x)$ to denote the open ball of radius $\epsilon$ around $x$.

Proposition 3.1. Let $(X, d)$ be a complete metric space and let $\mathcal{U}$ be an ultrafilter in $\operatorname{Ro}(X)$. Then either $\mathcal{U}$ defines a point in $X$, or there is some $\delta>0$ such that for every $x \in X$ there is $U \in \mathcal{U}$ such that $B_{\delta}(x) \cap U=\emptyset$.

Proof. We assume that the latter possibility fails and show that $\mathcal{U}$ defines a point in $X$. Assume that for every $n \in \omega$ there is some $x_{n} \in X$ such that $B_{2^{-n}}\left(x_{n}\right) \cap U \neq \emptyset$ for every $U \in \mathcal{U}$. Let $C_{n}=\operatorname{int}\left(\operatorname{cl}\left(B_{2^{-n}}\left(x_{n}\right)\right)\right) \in \operatorname{Ro}(X)$, so $C_{n} \cap U \neq \emptyset$ for every $U \in \mathcal{U}$. Since $\mathcal{U}$ is an ultrafilter in $\operatorname{Ro}(X)$, we have $C_{n} \in \mathcal{U}$ for every $n \in \omega$.

In particular, $C_{m} \cap C_{n} \neq \emptyset$ for every $m \leq n \in \omega$, so $d\left(x_{m}, x_{n}\right) \leq$ $2^{-m+1}$, which means that $\left(x_{n}\right)$ is a Cauchy sequence and hence converges 
to some $z \in X$. For every $n \in \omega, B_{2^{-n-2}}\left(x_{n+2}\right) \subseteq B_{2^{-n}}(z)$, and hence $U \cap B_{2^{-n}}(z) \neq \emptyset$. Since $z \in \operatorname{cl}(U)$ for every $U \in \mathcal{U}, \mathcal{U}$ defines $z$.

Proof of Theorem 1.1. For every $n \in \omega$ let $G_{n}$ be the subgroup of $G$ generated by $\bigcup_{x \in X} G\left|B_{1 / n}(x)\right|$, and let $H_{n}$ be the subgroup of $H$ generated by $\bigcup_{y \in Y} H\left|B_{1 / n}(y)\right|$. Observe that since $G$ is locally moving on $X$, every $G_{n}$ is also locally moving on $X$. Analogously every $H_{n}$ is locally moving on $Y$.

Now we define the following subsets $F_{X} \subseteq X$ and $F_{Y} \subseteq Y$ :

$F_{X}=\bigcup_{n \in \omega} \operatorname{Fix}\left(G_{n}\right) \cup \bigcup_{n \in \omega} \operatorname{Fix}\left(\varphi^{-1}\left(H_{n}\right)\right), F_{Y}=\bigcup_{n \in \omega} \operatorname{Fix}\left(H_{n}\right) \cup \bigcup_{n \in \omega} \operatorname{Fix}\left(\varphi\left(G_{n}\right)\right)$.

Let $F_{X}^{\prime}$ be the set of all $x \in X$ such that there is some ultrafilter $\mathcal{U}$ in $\operatorname{Ro}(X)$ which defines $x$ while $\psi(\mathcal{U})$ defines a point $y \in F_{Y}$. Let $\widetilde{X}=X \backslash\left(F_{X} \cup F_{X}^{\prime}\right)$ and $\widehat{X}=\{g(x) \mid g \in G, x \in \widetilde{X}\}$. Analogously, let $F_{Y}^{\prime}$ be the set of all $y \in Y$ such that there is some ultrafilter $\mathcal{U}$ in $\operatorname{Ro}(Y)$ which defines $y$ while $\psi^{-1}(\mathcal{U})$ defines a point $x \in F_{X}$. Let $\tilde{Y}=Y \backslash\left(F_{Y} \cup F_{Y}^{\prime}\right)$ and $\widehat{Y}=\{h(x) \mid h \in H$, $y \in \tilde{Y}\}$. It follows from the definition of $\widehat{X}$ and $\widehat{Y}$ that they are invariant under $G$ and $H$ respectively. Note that the definitions of $\widetilde{X}$ and $\tilde{Y}$ are symmetric, and so every claim we prove about $\widetilde{X}$ will be true for the same reason for $\widetilde{Y}$.

Claim 1. Let $\mathcal{U}$ be an ultrafilter in $\operatorname{Ro}(X)$ which defines some $x \in X \backslash F_{X}$. Then there is some $y \in Y$ such that $\psi(\mathcal{U})$ defines $y$.

Proof. Assume there is no $y \in Y$ such that $\psi(\mathcal{U})$ defines $y$. Then by Proposition 3.1 there is some $n \in \omega$ such that for every $y \in Y$ there is $U \in \mathcal{U}$ such that $B_{2^{-n}}(y) \cap \psi(U)=\emptyset$. Hence $H_{n} \subseteq H_{\psi(\mathcal{U})}$. So $\varphi^{-1}\left(H_{n}\right) \subseteq$ $\varphi^{-1}\left(H_{\psi(\mathcal{U})}\right)$, and thus from Corollary 2.3 we get $\varphi^{-1}\left(H_{n}\right) \subseteq G_{\mathcal{U}}$. Since $G_{\mathcal{U}} \subseteq G_{x}$ it follows that $\varphi^{-1}\left(H_{n}\right) \subseteq G_{x}$, which can't be since $x \notin F_{X}$ so $x \notin \operatorname{Fix}\left(\varphi^{-1}\left(H_{n}\right)\right)$.

Claim 2. Let $\mathcal{U}$ and $\mathcal{U}^{\prime}$ be ultrafilters in $\operatorname{Ro}(X)$ which both define some $x \in X \backslash F_{X}$. Then both $\psi(\mathcal{U})$ and $\psi\left(\mathcal{U}^{\prime}\right)$ define the same point in $Y$.

Proof. From the previous claim there are $y, y^{\prime} \in Y$ which are defined by $\psi(\mathcal{U})$ and $\psi\left(\mathcal{U}^{\prime}\right)$ respectively. Assume that $y \neq y^{\prime}$, and take some $n$ such that $1 / n \leq d\left(y, y^{\prime}\right) / 3$. For every $z \in Y$ we have either $y \notin \operatorname{cl}\left(B_{1 / n}(z)\right)$ or $y^{\prime} \notin \operatorname{cl}\left(B_{1 / n}(z)\right)$ (or both). So $H_{n} \subseteq H_{\psi(\mathcal{U}), \psi\left(\mathcal{U}^{\prime}\right)}$. Now, just as in the previous claim $\varphi^{-1}\left(H_{n}\right) \subseteq \varphi^{-1}\left(H_{\psi(\mathcal{U}), \psi\left(\mathcal{U}^{\prime}\right)}\right)$, and from Corollary 2.3 it follows that $\varphi^{-1}\left(H_{n}\right) \subseteq G_{\mathcal{U}, \mathcal{U}^{\prime}}$. Since both $\mathcal{U}$ and $\mathcal{U}^{\prime}$ define $x$, clearly $G_{\mathcal{U}, \mathcal{U}^{\prime}} \subseteq G_{x}$ and hence $\varphi^{-1}\left(H_{n}\right) \subseteq G_{x}$, which is a contradiction since $x \notin F_{X}$.

In light of the last two claims, we can define $\widetilde{\tau}$ for every $x \in \widetilde{X}$ to be the point in $Y$ defined by an ultrafilter $\psi(\mathcal{U})$ for some $\mathcal{U}$ which defines $x$. It 
follows from the last two claims and from the definition of $F_{X}^{\prime}$ and $F_{Y}^{\prime}$ that $\widetilde{\tau}$ is a well defined function, and that it is 1-1 and onto between $\widetilde{X}$ and $\widetilde{Y}$.

Claim 3. $\widetilde{\tau}(U \cap \tilde{X})=\psi(U) \cap \tilde{Y}$ for every $U \in \operatorname{Ro}(X)$.

Proof. Suppose $x \in U$, then $U \in \mathcal{U}$ for every ultrafilter $\mathcal{U}$ which defines $x$. Hence $\widetilde{\tau}(x) \in \operatorname{cl}(\psi(U))$ since $\widetilde{\tau}(x)$ is the point defined by $\psi(\mathcal{U})$. If $\widetilde{\tau}(x) \notin$ $\psi(U)$, then there is some ultrafilter $\mathcal{V}$ which defines $\widetilde{\tau}(x)$ and $-\psi(U) \in \mathcal{V}$. But then $-U \in \psi^{-1}(\mathcal{V})$ and $\psi^{-1}(\mathcal{V})$ defines $x$. Clearly $U \notin \mathcal{V}$ and this is a contradiction.

Since $\operatorname{Ro}(X)$ and $\operatorname{Ro}(Y)$ are bases for the topologies of $X$ and $Y$, the previous claim says that $\widetilde{\tau}$ is a bijection between the members of a base of $\widetilde{X}$ and the members of a base of $\widetilde{Y}$, so $\widetilde{\tau}$ is a homeomorphism.

Claim 4. $\widetilde{X}$ is co-meager in $X$. In particular, so is $\widehat{X}$.

Proof. Because of Corollary 2.4 and Proposition 2.5, both $F_{X}$ and $F_{Y}$ are meager. Now we show that for every $D \subseteq X \backslash F_{X}$, if $D$ is dense in some $U \in \operatorname{Ro}(X)$ then the set of points in $Y$ defined by ultrafilters $\psi(\mathcal{V})$ such that $\mathcal{V}$ defines some $x \in D$ is dense in $\psi(U)$. Take some $V \in \operatorname{Ro}(Y)$ such that $\operatorname{cl}(V) \subseteq \psi(U)$. So $\psi^{-1}(V) \subseteq U$. Let $z \in D \cap \psi^{-1}(V)$ and take some $\mathcal{V}$ which defines $z$. Clearly $\psi^{-1}(V) \in \mathcal{V}$ and so $V \in \psi(\mathcal{V})$. Since $z \notin F_{X}$ there is some $y \in Y$ defined by $\mathcal{V}$. Clearly $y \in \operatorname{cl}(V) \subseteq \psi(U)$ and we are done. It follows that $F_{X}^{\prime}$ is a countable union of nowhere dense sets.

Notice that for every $g \in G$, the function $\varphi(g) \circ \widetilde{\tau} \circ g^{-1}$ is a homeomorphism between $g(\widetilde{X})$ and $\varphi(g)(\widetilde{Y})$.

Claim 5. $\varphi(g) \circ \widetilde{\tau} \circ g^{-1}(U \cap g(\tilde{X}))=\psi(U) \cap \varphi(g)(\tilde{Y})$ for all $U \in \operatorname{Ro}(X)$.

Proof. We have $\varphi(g) \circ \widetilde{\tau} \circ g^{-1}(U \cap g(\widetilde{X}))=\varphi(g) \circ \widetilde{\tau}\left(g^{-1}(U) \cap \widetilde{X}\right)=$ $\varphi(g)\left(\psi\left(g^{-1}(U)\right) \cap \widetilde{Y}\right)=\psi(U) \cap \varphi(g)(\widetilde{Y})$. The second equality follows from Claim 3, and the third from the fact that $\psi$ induces $\varphi$.

Now we can define $\tau=\bigcup_{g \in G} \varphi(g) \circ \widetilde{\tau} \circ g^{-1}$. Since $\widetilde{\tau}$ is a homeomorphism, Claim 5 shows that $\tau$ is also a homeomorphism. From the definition of $\tau$ it follows that $\widehat{X}$ is its domain and $\widehat{Y}$ its range. Claim 4 and the fact that $\psi$ induces $\varphi$ imply that $\tau$ induces $\varphi$. So we are done.

Proof of Theorem 1.2. Let $\tau$ be as promised by Theorem 1.1. We only need to show that $\operatorname{Dom}(\tau)=X$ and $\operatorname{Rng}(\tau)=Y$. Clearly $X \backslash \operatorname{Dom}(\tau)$ is closed under $G$ since $\operatorname{Dom}(\tau)$ is, and meager since $\operatorname{Dom}(\tau)$ is co-meager. So, the orbits of $G$ being of the second category, we have $X \backslash \operatorname{Dom}(\tau)=\emptyset$ and thus $\operatorname{Dom}(\tau)=X$. For the same reason $\operatorname{Rng}(\tau)=Y$.

Proof of Corollary 1.3 . The fact that $G$ is strongly locally homogeneous on $X$ implies it is locally moving on $X$. Moreover, it implies that $G(x)$ 
contains an open subset of $X$ for every $x \in X$, and thus is of the second category in $X$. Since the same holds for $H$ and $Y$, the corollary follows from Theorem 1.2 .

\section{References}

[Ru1] M. Rubin, On the reconstruction of topological spaces from their groups of homeomorphisms, Trans. Amer. Math. Soc. 312 (1989), 487-537.

[Ru2] -, Locally moving groups and reconstruction problems, in: Ordered Groups and Infinite Permutation Groups, Math. Appl. 354, Kluwer, Dordrecht, 1996, 121-151.

[vM] J. van Mill, Homeomorphism groups and homogeneous spaces, Proc. Amer. Math. Soc. 92 (1984), 449-453.

Edmund Ben-Ami

Department of Mathematics

Ben Gurion University of the Negev

Beer Sheva, Israel

E-mail: bae@cs.bgu.ac.il

Received 13 August 2008;

in revised form 11 November 2009 